UDC 544.022.341, 544.022.382

\title{
DIFFUSION CHARACTERISTICS OF RADIATION DEFECTS IN IRON: MOLECULAR DYNAMICS DATA
}

\author{
A.B. Sivak, D.N. Demidov, P.A. Sivak
}

\author{
NRC «Kurchatov Institute», Moscow, Russia
}

Temperature dependences of the diffusion characteristics of radiation defects, namely, clusters of up to five self-interstitial atoms (SIAs), were studied by the molecular dynamics method for bcc Fe at temperatures in the range of 300-1200 K. The above-mentioned diffusion characteristics included the diffusion coefficient, the tracer correlation factor, the mean distance traveled between changes in the migration direction, the frequency of migration direction changes, etc. The activation energy values for diffusion and changes in the migration direction were determined for the defects of interest for different temperature ranges. The temperature and size dependences of the SIA cluster diffusion mechanism (1D vs 3D) and their potential implications for the microstructure of materials exposed to irradiation are discussed.

Key words: iron, molecular dynamics, interatomic interaction potentials, radiation defects, diffusion mechanisms, diffusivities.

DOI: $10.21517 / 0202-3822-2021-44-2-148-157$

\section{ДИФФУЗИОННЫЕ ХАРАКТЕРИСТИКИ РАДИАЦИОННЫХ ДЕФЕКТОВ В ЖЕЛЕЗЕ: МОЛЕКУЛЯРНО-ДИНАМИЧЕСКИЕ ДАННЫЕ}

\author{
А.Б. Сивак, Д.Н. Демидов, П.А. Сивак
}

НИЦ «Курчатовский институт», Москва, Россия

\begin{abstract}
Методом молекулярной динамики исследованы температурные зависимости диффузионных характеристик радиационных дефектов - кластеров собственных межузельных атомов (СМА), содержащих до пяти СМА — в температурном диапазоне 300 - 1200 К в ОЦК Fe. Упомянутые диффузионные характеристики включали в себя коэффициент диффузии, корреляционный множитель меченых атомов, среднюю длину диффузионного пробега до смены направления диффузии, частоту смен направлений диффузии, др. Определены значения энергии активации диффузии и энергии активации смен направлений диффузии для рассмотренных типов дефектов для разных температурных диапазонов. Обсуждаются зависимости механизма диффузии кластеров CMA (1D vs 3D) от температуры и размера кластеров и их возможные следствия для эволюции микроструктуры материала под облучением.
\end{abstract}

Ключевые слова: железо, молекулярная динамика, потенциалы межатомных взаимодействий, радиационные дефекты, механизмы диффузии, коэффициенты диффузии.

\section{INTRODUCTION}

The diffusion of radiation defects to sinks (dislocations, grain boundaries, sub-boundaries, interfaces, etc.) and their subsequent absorption affect the microstructure evolution of fusion/fission reactor structural materials with resultant changes in their physical and mechanical properties (radiation creep and embrittlement, and void swelling) [1]. The physical modelling of changes in material properties induced by irradiation or mechani$\mathrm{cal} /$ thermal loads requires the characteristics of radiation defects, to be used as inputs for such models.

Damaging neutron irradiation causes atomic collision cascades, which generate not only self-point defects (SPDs) but their clusters as well [2]. A molecular dynamics (MD) study of primary radiation damage in iron [3, 4] has shown that the higher the cascade damage energy, the greater is the tendency for self-interstitial atoms (SIAs) to form clusters: the fraction of single SIAs decreases from 70 to $40 \%$ with increase in the damage energy from 1 to $50 \mathrm{keV}$. In a given range of damage energies, $30 \%$ to $40 \%$ of surviving SIAs are grouped in clusters of two to five.

Because the properties of SIAs and their small clusters are difficult to assess experimentally due to small spatial sizes $(\sim \mathrm{nm})$, computer simulation is actively used, which allowed to determine the diffusion characteristics of single SIAs and clusters of up to 91 SIAs in Fe [5-18]. The results of computer simulations are highly sensitive to the selection of applicable interatomic interaction potential. Elastic moduli determined experimentally for $\mathrm{Fe}$ and $\mathrm{V}$ were very much at variance with values calculated based on the density functional theory (DFT) [19]. Therefore, the applicability of DFT to the calculation of SPDs is questionable, as is the use of potentials with parameters fitted to DFT data. In this context, data from many publications cited above require validation and refinement using a reliable interatomic interaction potential, consistent with experimental data. It was shown in [19] that the interatomic interaction potential R01 [20-23], developed for the bcc metal Fe, agreed well with the experimentally measured crystal bulk properties (elastic constants at $0 \mathrm{~K}$ and the temperature dependence of the lattice parameter), the properties of SIAs and vacancies, as well as threshold displacement energies, and was therefore recommended for use in MD simulations of atomic collision cascades and SPDs. Using R01, the crystallographic, energetic, and diffusion properties of SIAs $[8,12,13]$ and di-interstitials $[9,17,18]$ were determined and atomic collision cascades were simulated $[3,4]$. 
This paper describes the results of the study of the diffusion characteristics of clusters of three to five SIAs in bcc Fe at temperatures in the range of 300-1200 K, performed by MD using the R01 potential. The temperature and size dependences of the SIA cluster diffusion mechanism (1D vs 3D) and their potential implications for the microstructure of materials exposed to irradiation are discussed.

\section{SIMULATION TECHNIQUE}

Cluster size $q$ is the number of SIAs in a cluster. An SIA cluster of size $q$ is denoted as $q$-SIA. To study the diffusion of SIA clusters with $q=3-5$, MD simulations were carried out using cubic model crystallites with periodic boundary conditions. Those crystallites represented a microcanonical ensemble. The lattice constant, $a$, was chosen to ensure that pressure $P$ in the crystallite bulk was zero (accurate to $0.1 \mathrm{eV} / \mathrm{nm}^{3}$ ) at given temperature $T$. The equations of motion were integrated by the Verlet algorithm [24]. The integration time step was selected to ensure that the average of atomic displacements per iteration was $\sim 0.005 a$. The positions of clustered SIAs were determined from the numbers of atoms in Wigner-Seitz cells (WSCs). The position of the WSC closest to the cluster mass centre was taken as the position of the SIA cluster. The model crystallite size was chosen so that the boundary condition effect on the calculation results was insignificant. Crystallites with a side length of $13 a$ (the total number of atoms was $4394+3$ and $4394+4$, respectively) were used to simulate the diffusion of 3-SIA and 4-SIA clusters. For 5-SIA clusters, the side length was $15 a(6750+5)$.

The most stable SIA cluster configurations were chosen as initial configurations. Table 1 shows their formation and binding energies ( $E^{F}$ and $E^{B}$, respectively) for clusters of two to five SIAs (calculated in [9]), as well as their schematic spatial configurations (viewed from different perspectives). All SIA clusters with $q>2$ are sets of crowdions or dumbbells split in the $\langle 111\rangle$ direction (henceforth, the split direction will be referred to as cluster orientation). SIAs occupy the most compact set of adjacent close-packed directions projected onto the plane normal to the splitting direction (see Table 1).

T a b 1 e 1 . Crystallographic configurations of simulated SIA clusters in Fe, their formation energies $\boldsymbol{E}^{\boldsymbol{F}}$ and binding energies $\boldsymbol{E}^{\boldsymbol{B}}$

\begin{tabular}{|c|c|c|}
\hline Cluster type & Crystallographic configuration & Formation energy and binding energy, eV \\
\hline 2-SIA & & $\begin{array}{l}E^{F}=7.884 \\
E^{B}=0.884\end{array}$ \\
\hline 3-SIA & & $\begin{array}{l}E^{F}=11.04 \\
E^{B}=1.230\end{array}$ \\
\hline 4-SIA & & $\begin{array}{l}E^{F}=13.37 \\
E^{B}=2.050\end{array}$ \\
\hline 5-SIA & & $\begin{array}{l}E^{F}=15.95 \\
E^{B}=1.810\end{array}$ \\
\hline
\end{tabular}


For each given cluster size and temperature, one hundred diffusion trajectories were obtained. Table 2 lists the total physical simulation time and the number of cluster jumps summed over all trajectories for clusters and temperatures of interest. To calculate the diffusivity $D^{\mathrm{d}}$ of an SIA cluster, each simulated diffusion trajectory was split into several isochronous segments of duration $\tau$. The choice of the $\tau$ value is a trade-off between the need to have a large enough number of segments (to minimize random errors) and a large enough number of defect jumps at each of those segments to account for any significant spatial correlation associated with SIA cluster diffusion (to minimize systematic errors). The empirical dependence $\tau[\mathrm{ps}]=0.66 \exp (-\beta \cdot 0.078 \mathrm{eV})$ with $\beta=\left(k_{\mathrm{B}} T\right)^{-1}$ obtained in [18] for 2-SIAs, was used in this study to select $\tau$ for a given $T$. Our analyses proved that it is applicable to clusters of three to five SIAs. For all isochronous segments of all trajectories, values $r^{2} / 6 \tau$ were calculated, where $r$ is the displacement of the SIA cluster during time $\tau$ for one segment. The value $D^{\mathrm{d}}$ was determined as the average of these values, and the $D^{\mathrm{d}}$ inaccuracy was estimated as the standard error for the specified set.

$\mathrm{T} \mathrm{a} \mathrm{b} 1$ e 2. Physical simulation time $t_{\mathrm{s}}$ and the number of jumps $j_{\mathrm{s}}$ for the discussed types of clusters in Fe (integral over all trajectories)

\begin{tabular}{|c|c|c|c|c|c|c|}
\hline \multirow{2}{*}{$T, \mathrm{~K}$} & \multicolumn{2}{|c|}{ 3-SIA } & \multicolumn{2}{|c|}{ 4-SIA } & \multicolumn{2}{|c|}{ 5-SIA } \\
\hline & $t_{\mathrm{s}}, \mu \mathrm{s}$ & $j_{\mathrm{s}}$ & $t_{\mathrm{s}}, \mu \mathrm{s}$ & $j_{\mathrm{s}}$ & $t_{\mathrm{s}}, \mu \mathrm{s}$ & $j_{\mathrm{s}}$ \\
\hline 300 & 61.5 & $2.87 \cdot 10^{7}$ & 122 & $2.43 \cdot 10^{8}$ & 70.9 & $1.89 \cdot 10^{8}$ \\
\hline 400 & 34.7 & $1.80 \cdot 10^{7}$ & 65.7 & $1.50 \cdot 10^{8}$ & 47.1 & $1.42 \cdot 10^{8}$ \\
\hline 500 & 21.1 & $1.20 \cdot 10^{7}$ & 33.1 & $8.38 \cdot 10^{7}$ & 27.2 & $9.07 \cdot 10^{7}$ \\
\hline 700 & 15.0 & $9.47 \cdot 10^{6}$ & 17.1 & $4.72 \cdot 10^{7}$ & 18.7 & $6.76 \cdot 10^{7}$ \\
\hline 800 & 8.81 & $5.97 \cdot 10^{6}$ & 8.84 & $2.62 \cdot 10^{7}$ & 12.3 & $4.76 \cdot 10^{7}$ \\
\hline 900 & 5.23 & $3.80 \cdot 10^{6}$ & - & - & 5.94 & $2.44 \cdot 10^{7}$ \\
\hline 1000 & 2.63 & $2.00 \cdot 10^{6}$ & 1.05 & $3.91 \cdot 10^{6}$ & 3.24 & $1.40 \cdot 10^{7}$ \\
\hline 1100 & 1.34 & $1.08 \cdot 10^{6}$ & - & - & - & - \\
\hline 1200 & 0.556 & $4.73 \cdot 10^{5}$ & 0.126 & $4.87 \cdot 10^{5}$ & 1.22 & $5.52 \cdot 10^{6}$ \\
\hline
\end{tabular}

Tracer diffusivity $D^{\text {tr }}$ (self-diffusivity per one SIA) was calculated by Einstein-Smoluchowski relation [25] using the initial and end positions of each atom in crystallite models (100 program runs). For each dataset, selfdiffusivity intermediate values were calculated. The final $D^{\text {tr }}$ value was obtained by their averaging. The $D^{\text {tr }}$ inaccuracy was estimated as the standard error over the specified set of 100 intermediate values.

Tracer correlation factor $f^{\mathrm{tr}}$ was determined as the ratio of $D^{\mathrm{tr}}$ to $D^{\mathrm{d}}$. Because $f^{\mathrm{tr}}$ is affected by the cluster diffusion mechanism, it can be used to indicate changes in the mechanism of SIA clusters diffusion. E.g., in the case of a one-dimensional (1D) diffusion of a crowdion, $f^{\mathrm{tr}}=0$ [26], whereas in the case of a 3D diffusion of $\langle 110\rangle$ dumbbells under Johnson's mechanism, $f^{\text {tr }}=0.4151 \pm 0.0001$ [18]. Usually, cluster diffusion is promoted by a mixed mechanism: clusters make a number of jumps in one of the $\langle 111\rangle$ directions, followed by a change in the migration direction, resulting from the cluster reorientation.

Other metrics that allow the contributions of the 1D/3D diffusion mechanisms to be assessed are the mean distance traveled between changes in the migration direction [27, 28], and the frequency of migration direction changes ( $l_{\text {ch }}$ and $v^{\mathrm{R}}$, respectively). To determine $v^{\mathrm{R}}$ and $l_{\text {ch }}$, a special algorithm analyzing MD defect trajectories was developed. In this study, $l_{\mathrm{ch}}$ is expressed as $l_{\mathrm{ch}}=\left(6 D^{\mathrm{d}}\left\langle\Delta t_{1 \mathrm{D}}\right\rangle\right)^{1 / 2}$, where $\left\langle\Delta t_{1 \mathrm{D}}\right\rangle$ is the average time between two successive reorientations.

The instants of time, at which the cluster reorientations occurred were determined using the following algorithm:

- (1) construct four cylinders of radius $\rho$ with axes oriented along four close-packed directions $\langle 111\rangle$ and passing through the initial position of the SIA cluster on the diffusion trajectory;

- (2) determine four instants of time, at which the trajectory leaves each cylinder;

- (3) the latest of the four instants of time, identified in step 2, is taken as the instant, at which the cluster is reoriented;

- (4) construct cylinders of radius $\rho$ with axes oriented along $\langle 111\rangle$ and passing through the cluster's new position at the instant the last cluster reorientation is detected;

- (5) repeat steps $2-4$ until the last cluster reorientation on the diffusion trajectory is detected. 
Upon the completion of this algorithm, the durations $\Delta t_{1 \mathrm{D}}$ between successive moments of cluster reorientations are determined and averaged to obtain the desired $\left\langle\Delta t_{1 \mathrm{D}}\right\rangle$ value. Inaccuracy is determined as the standard error for the entire set of $\Delta t_{1 \mathrm{D}}$ values.

Radius $\rho$ was an algorithm parameter obtained using an iterative selection method, in which an initially large value (e.g., $5 a$ ) was successively decreased by $a / 2$ until it stopped to produce any noticeable effect on the total number of detected 1D segments and the final values of $l_{\mathrm{ch}}$ and $\left\langle\Delta t_{1 \mathrm{D}}\right\rangle$. The obtained values of $\rho$ are $1.5 a$ for 1-, 2-SIA and $2 a$ for 3-, 4-, 5-SIA.

Frequency $v^{\mathrm{R}}$ was determined as the reciprocal of $\left\langle\Delta t_{1 \mathrm{D}}\right\rangle$. Since the time intervals between two successive reorientations have a Poisson distribution, the relative error $\delta v_{\mathrm{R}}$ for the frequency of reorientations $v^{\mathrm{R}}$ can be determined as $N_{\mathrm{R}}^{-1 / 2}$, where $N_{\mathrm{R}}$ is the total number of reorientations detected at a given temperature. The $\delta v_{\mathrm{R}}$ values at different temperatures vary from $1 \%$ to $50 \%$. The least accurate $v^{\mathrm{R}}$ values were obtained at temperatures below $500 \mathrm{~K}$ since reorientations were rare and their total number for the entire simulation period was usually $\sim 10$ (for all 100 diffusion trajectories at a given $T$ ).

\section{RESULTS AND DISCUSSION}

Figs. 1, 2 show typical MD trajectories of single and clustered SIAs at temperatures of 300, 700 and $1200 \mathrm{~K}$, as well as the physical simulation time for each illustrated trajectory $\tau_{\mathrm{m}}$. Data for single SIAs and 2-SIA clusters were obtained in $[8,12,13]$ and $[17,18]$, respectively. The diffusion of single SIAs and 2-SIA clusters has a much higher degree of three-dimensionality compared with larger clusters.
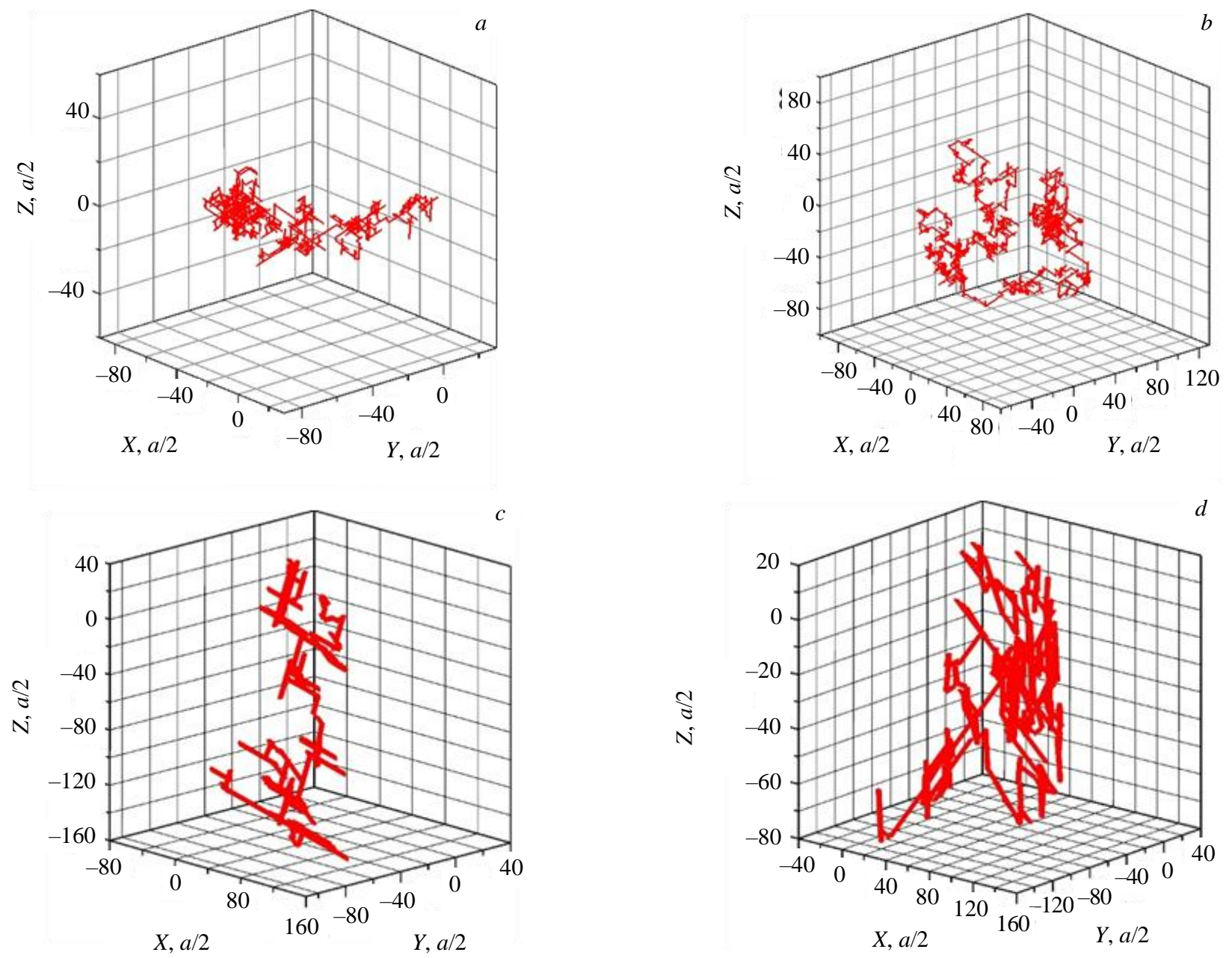

Fig. 1. MD trajectories of single SIAs $(a, b)$ and 2-SIA $(c, d)$ clusters at $300 \mathrm{~K}(a, c)$ and $700 \mathrm{~K}(b, d), \tau_{\mathrm{m}}: 65.4(a), 2.97(b), 7.74(c), 4.12 \mathrm{~ns}(d)$ 

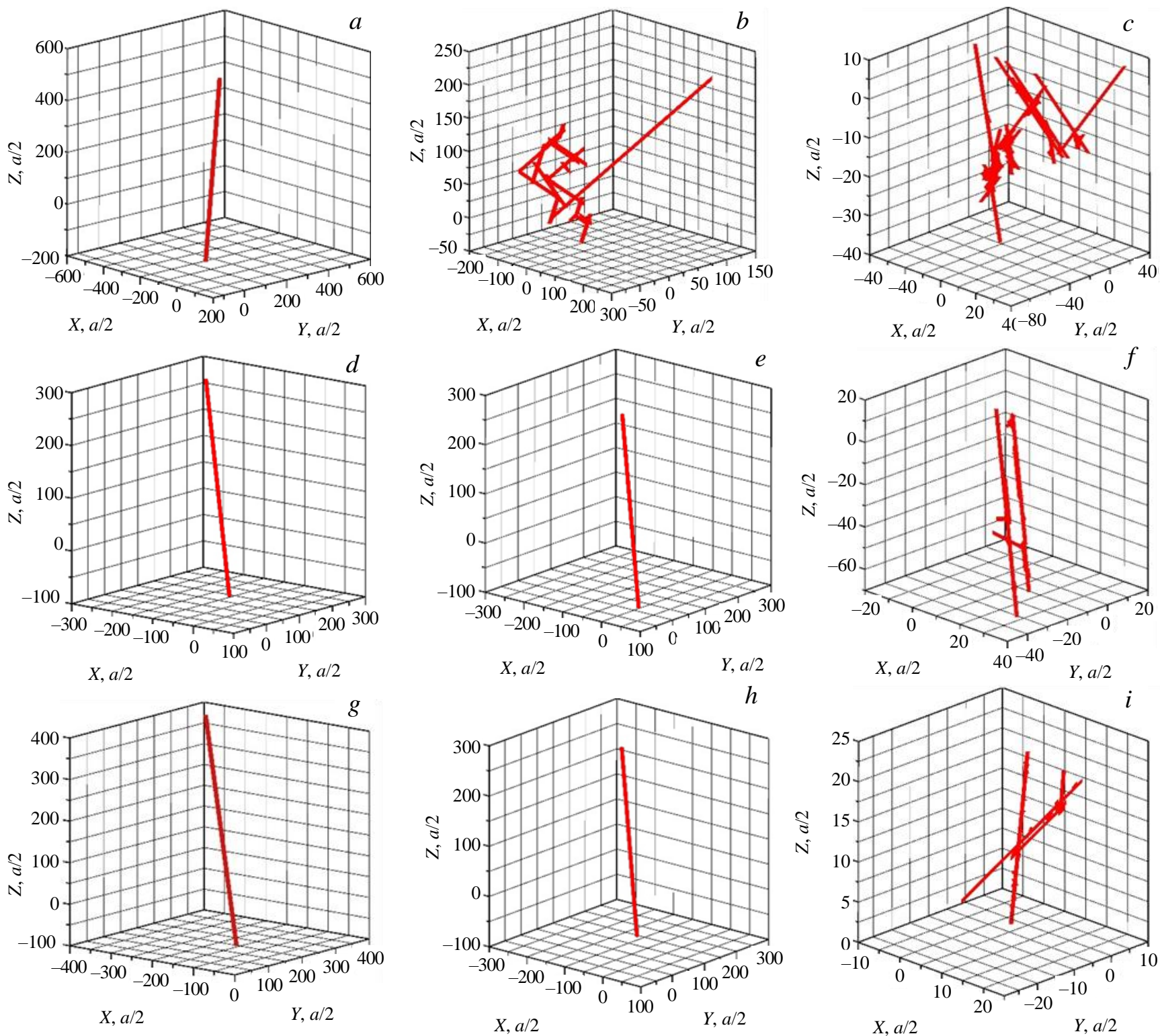

Fig. 2. MD trajectories of $q$-SIAs, $q=3(a, b, c), 4(d, e, f), 5(g, h, i)$ at $300(a, d, g), 700(b, e, h), 1200 \mathrm{~K}(c, f, i), \tau_{\mathrm{m}}=25.6(a), 9.52(b)$, $5.21(c), 160(d), 17.1(e), 0.422(f), 11.0(g), 12.4(h), 0.297 \mathrm{~ns}(i)$

Fig. 3 shows the calculated temperature dependences of diffusivity $D^{\mathrm{d}}$ and tracer correlation factor $f^{\text {tr }}$ for $q$-SIAs $(q=1-5)$.
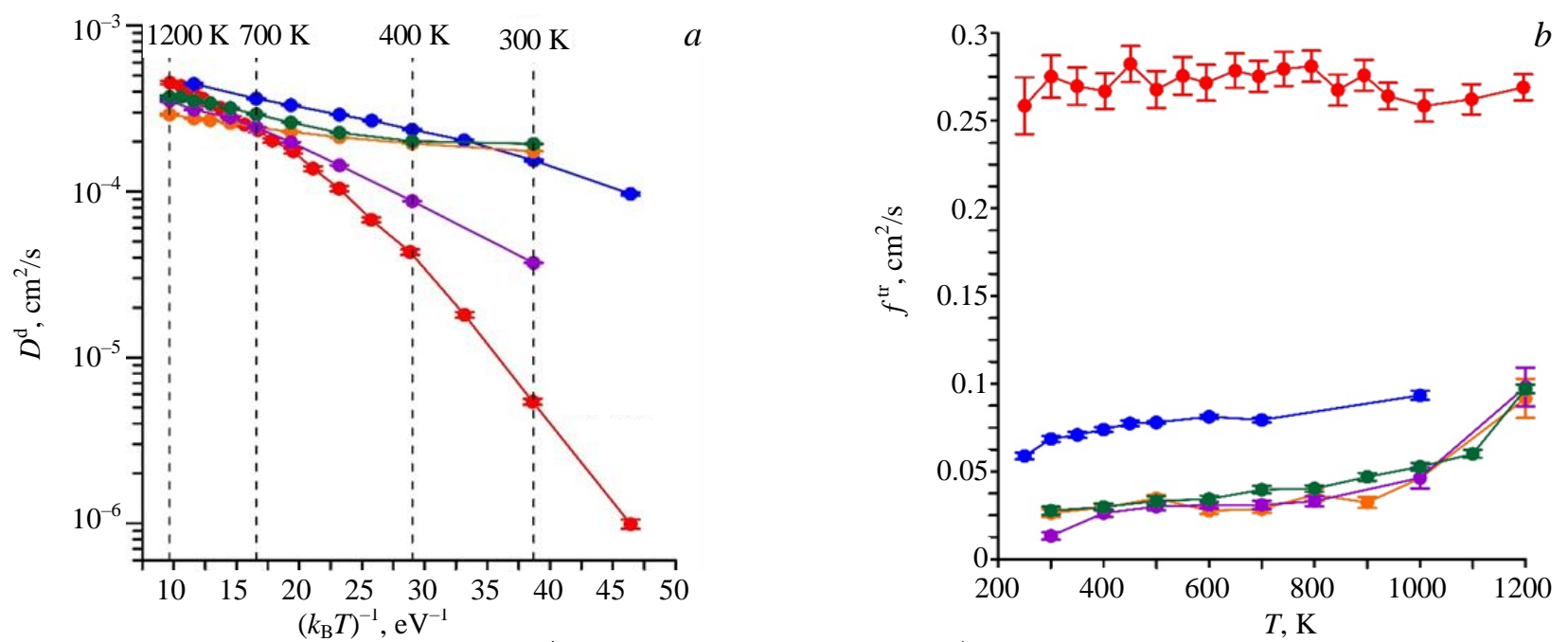

Fig 3. Temperature dependences of diffusivities $D^{\mathrm{d}}(a)$ and tracer correlation factor $f^{\mathrm{tr}}(b)$ for $q$-SIAs $(q=1-5)$ in Fe: $\bullet-1$-SIA, $\bullet-$ 2-SIA, $\bullet$ 3-SIA, $\bullet$ 4-SIA, - 5-SIA 
Values $D^{\mathrm{d}}$ for the discussed defects differ slightly at high temperatures, with the largest difference (30\%) observed between 2-SIA and 5-SIA clusters at $1000 \mathrm{~K}$. This difference is much more significant at low temperatures (e.g., the diffusivities of single SIAs and those of 3-SIA clusters differ by a factor of 36 at $300 \mathrm{~K}$ ). 3-SIA clusters show the greatest diffusivity among all defect types under review at $T<350 \mathrm{~K}$. The temperature dependences of $D^{\mathrm{d}}$ are not linear in Arrhenius coordinates (Fig. 3, a). Therefore, an accurate description of MD data can be obtained by Arrhenius dependences only for particular temperature ranges.

Table 3 lists the fitting parameters of such dependences (preexponential factor $D_{0}$ and diffusion activation energy $E^{\mathrm{AD}}$ ) with indication of temperature ranges, for which those parameters have been obtained. In the case of 3- and 5-SIA clusters, $D^{\mathrm{d}}$ is weakly dependent on $T$ at $T \leq 400 \mathrm{~K}\left(E^{\mathrm{AD}} \leq 0.01 \mathrm{eV}\right)$. The low $E^{\mathrm{AD}}$ value for 3-SIA clusters $(0.004 \mathrm{eV})$ is consistent with the result of direct molecular statics calculations [9] of the energy barrier for cluster migration, which is less than $10^{-4} \mathrm{eV}$. At $T \geq 700 \mathrm{~K}$, the $E^{\mathrm{AD}}$ value for the clusters under review is $2-4$ times lower than that for a single SIA (see Table 3).

$\mathrm{T}$ a b 1 e 3 . Arrhenius approximation parameters for $D^{\mathrm{d}}(T)$ for $q$-SIA $(q=1-5)$ in Fe

\begin{tabular}{|c|c|c|c|c|c|c|}
\hline \multirow[b]{2}{*}{$q$} & \multicolumn{3}{|c|}{ Low temperature approximation } & \multicolumn{3}{|c|}{ High temperature approximation } \\
\hline & $D_{0}, \mathrm{~cm}^{2} / \mathrm{s}$ & $E^{\mathrm{AD}}, \mathrm{eV}$ & $T$ range, $\mathrm{K}$ & $D_{0}, \mathrm{~cm}^{2} / \mathrm{s}$ & $E^{\mathrm{AD}}, \mathrm{eV}$ & $T$ range, $\mathrm{K}$ \\
\hline 1 & $2.59 \cdot 10^{-2}$ & 0.219 & $250-350$ & $1.25 \cdot 10^{-3}$ & 0.101 & $600-1200$ \\
\hline 2 & $1.33 \cdot 10^{-3}$ & 0.056 & $250-350$ & $6.43 \cdot 10^{-4}$ & 0.034 & $400-800$ \\
\hline 3 & $2.25 \cdot 10^{-4}$ & 0.004 & $300-400$ & $5.38 \cdot 10^{-4}$ & 0.036 & $700-1200$ \\
\hline 4 & $1.03 \cdot 10^{-3}$ & 0.086 & $300-700$ & $5.72 \cdot 10^{-4}$ & 0.051 & $700-1200$ \\
\hline 5 & $2.82 \cdot 10^{-4}$ & 0.013 & $300-500$ & $3.70 \cdot 10^{-4}$ & 0.025 & $600-1200$ \\
\hline
\end{tabular}

The $E^{\mathrm{AD}}$ values obtained in this study are very much at variance with data reported in [6], where $E^{\mathrm{AD}}=0.024 \pm 0.004 \mathrm{eV}$ regardless of cluster size. The potential used in [6] was developed by Osetsky et al. [29]. The most stable SIA configuration allowing for that potential is $\langle 111\rangle$ dumbbell, which does not agree with experimental data [30].

As one can see from Fig. 3, $b$, the one-dimensionality of the SIA clusters' diffusion becomes much more pronounced as the cluster size, $q$, increases from 1 to 3 ( $f^{\text {tr }}$ decreases from $\sim 0.28$ to $0.03-0.08$ depending on the temperature), while the $f^{\text {tr }}$ value becomes almost independent of $q$ at $q \geq 3$. The $f^{\text {tr }}$ value does not go to zero even where the clusters' diffusion is almost completely one-dimentional (as in the case of a 5-SIA cluster at $300 \mathrm{~K}$ ). The reason for $f^{\mathrm{tr}} \neq 0$ is that atoms forming a SIA cluster may move from their original positions in the lattice to neighboring close-packed rows. Therefore, even if the cluster's mass centre moves one-dimensionally all the time, the cluster is unable to completely «cover up the footprints», the result being the non-zero $D^{\mathrm{tr}}$ and $f^{\mathrm{tr}}$. The inability of $f^{\text {tr }}$ to take the zero value is almost entirely determined by such changes of atomic positions within a $q$-SIA cluster, where $q \geq 3$. Incidentally, $f^{\text {tr }}$ does not characterize the diffusion mechanism of such clusters. In this context, the $f^{\text {tr }}$ value is useless for the identification of the cluster diffusion mechanism, and other characteristics are called for.

Fig. 4 shows the temperature dependences of the frequency of changes in the migration direction and the average distance traveled between migration direction changes for $q$-SIAs $(q=1-5)$ in Fe. The inaccuracy of
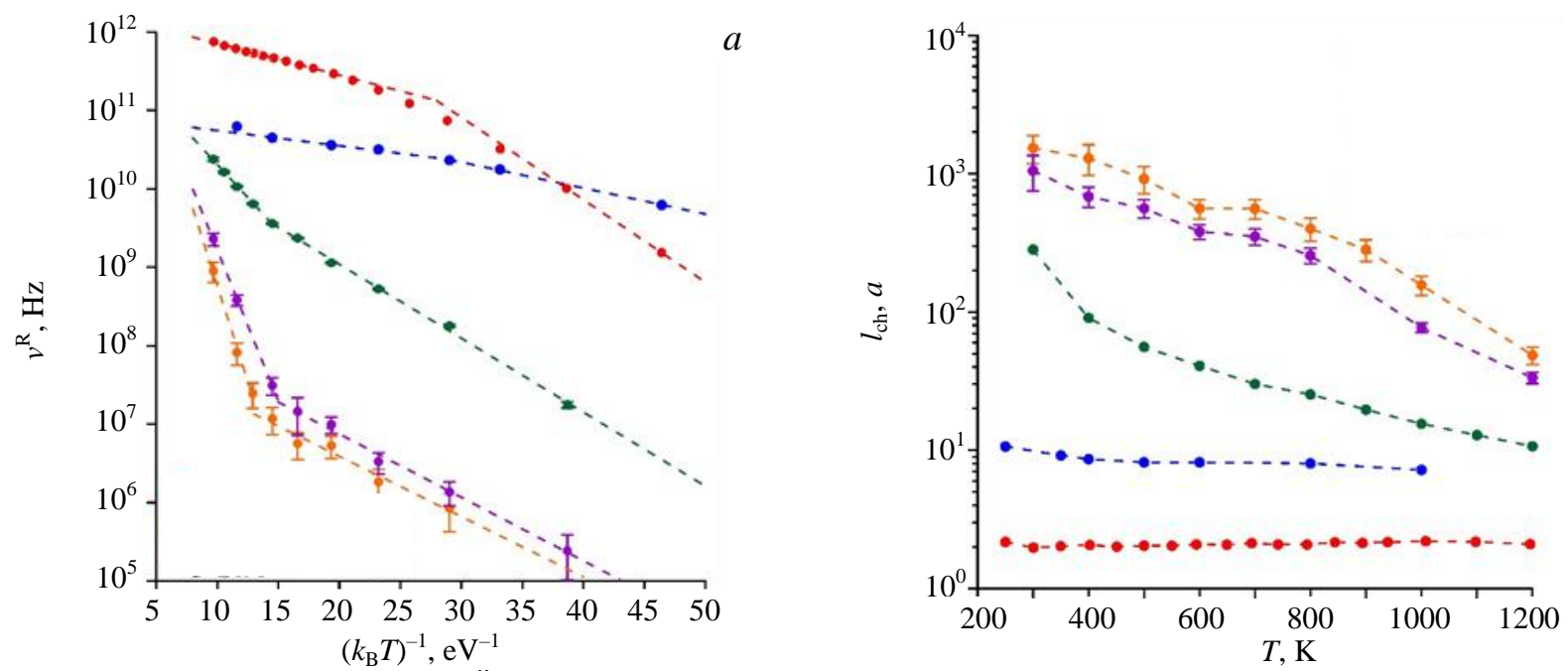

Fig. 4. Temperature dependences of $v^{\mathrm{K}}(a)$ and $l_{\mathrm{ch}}(b)$ for $q$-SIAs $(q=1-5)$ in Fe: $\bullet-1$-SIA, $\bullet-2$-SIA, $\bullet-3$-SIA, $\bullet-$ 4-SIA, - 5-SIA 
$v^{\mathrm{R}}$ and $l_{\text {ch }}$ is not indicated in the plots, if smaller than the size of the points. Both $v^{\mathrm{R}}$ and $l_{\text {ch }}$, in contrast to $f^{\text {tr }}$, are much dependent on $q$ across the whole range of $q$ values under review.

The temperature dependences of $v^{R}$ are not linear in Arrhenius coordinates (Fig. 4, $a$ ); therefore, an accurate description of the MD data can only be obtained by Arrhenius dependences for particular temperature ranges. Table 4 lists the fitting parameters of such dependences (preexponential factor $v_{0}$ and reorientation activation energy $E^{\mathrm{R}}$ ) with indication of temperature ranges, for which those parameters have been obtained. At low temperatures, the $E^{\mathrm{R}}$ values for $q$-SIA clusters with $q \geq 3$ are close to each other and equal $\sim 0.2 \mathrm{eV}$. At high temperatures, $E^{\mathrm{R}}$ becomes $2-6$ times higher (the higher $q$, the higher the difference) suggesting the roles of different mechanisms in cluster reorientations at low and high temperatures.

$\mathrm{T}$ a $\mathrm{b} l \mathrm{l}$ 4. Arrhenius approximation parameters for $\boldsymbol{v}^{\mathrm{R}}(\boldsymbol{T})$ for $\boldsymbol{q}$-SIAs $(\boldsymbol{q}=\mathbf{1}-\mathbf{5})$ in Fe

\begin{tabular}{c|c|c|c|c|c|c}
\hline \multirow{2}{*}{$q$} & \multicolumn{3}{|c|}{ Low temperature approximation } & \multicolumn{3}{c}{ High temperature approximation } \\
\cline { 2 - 6 } & $v_{0}, \mathrm{~Hz}$ & $E^{\mathrm{R}}, \mathrm{eV}$ & $T$ range, $\mathrm{K}$ & $v_{0}, \mathrm{~Hz}$ & $E^{\mathrm{R}}, \mathrm{eV}$ & $T$ range, $\mathrm{K}$ \\
\hline 1 & $1.16 \cdot 10^{14}$ & 0.242 & $250-300$ & $1.81 \cdot 10^{12}$ & 0.093 & $600-1200$ \\
2 & $2.16 \cdot 10^{11}$ & 0.076 & $250-400$ & $8.67 \cdot 10^{10}$ & 0.045 & $400-800$ \\
3 & $8.38 \cdot 10^{10}$ & 0.217 & $300-800$ & $1.01 \cdot 10^{12}$ & 0.390 & $800-1200$ \\
4 & $3.11 \cdot 10^{8}$ & 0.186 & $300-700$ & $1.20 \cdot 10^{13}$ & 0.888 & $800-1200$ \\
5 & $1.35 \cdot 10^{8}$ & 0.177 & $300-800$ & $4.50 \cdot 10^{13}$ & 1.125 & $900-1200$ \\
\hline
\end{tabular}

The $l_{\text {ch }}$ value for single SIAs becomes temperature independent in the temperature range of $250-1200 \mathrm{~K}$. Its average value $(2.1 a)$ is consistent with the temperature independent $f^{\text {tr }}$ for the corresponding temperature range (see Fig. $3, b$ ). For 2-SIA clusters, $l_{\mathrm{ch}}$ decreases from $10.6 a$ to $7.2 a$ with temperature rising from $250 \mathrm{~K}$ to $1000 \mathrm{~K}$, respectively, which is also consistent with the $f^{\text {tr }}$ increase. For clusters with $q \geq 3, l_{\text {ch }}$ decreases dozens of times with temperature rising from $300 \mathrm{~K}$ to $1200 \mathrm{~K}$ (from $283 a$ to $10.7 a$, from $1051 a$ to $33.5 a$, from $1535 a$ to $48.7 a$ for clusters with $q=3,4,5$, respectively).

The sink strengths of various microstructure elements are sensitive to the dimensionality of radiation defects' diffusion (sink strengths for 1D and 3D diffusion types may differ by several orders of magnitude) [27]. For example, sink strength $k^{2}$ of spherical absorbers with radius $R$ and number density $N$ (the number of absorbers per unit volume) is

$$
k_{3 \mathrm{D}}^{2} \approx 4 \pi R N \text { for a 3D diffusion }[31,32] \text {, }
$$

and is

$$
k_{1 \mathrm{D}}^{2}=6\left(\pi R^{2} N\right)^{2} \text { for a } 1 \mathrm{D} \text { diffusion [33]. }
$$

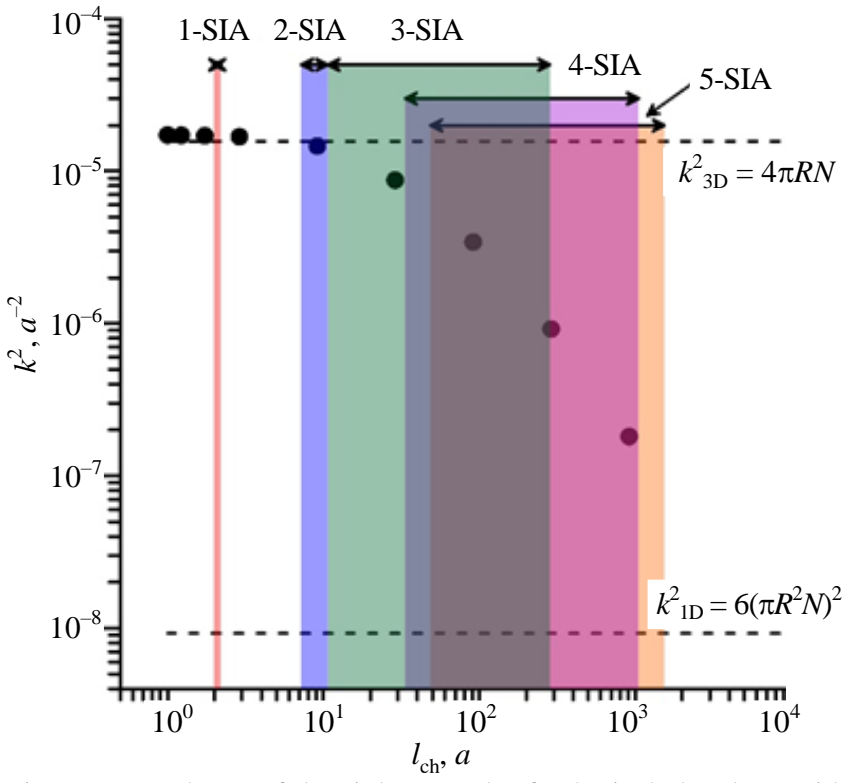

Fig. 5. Dependence of the sink strength of spherical absorbers with radius $R=10 a$ and number density $N=(200 a)^{-3}$ on the average distance traveled between migration direction changes, $l_{\mathrm{ch}}$. The color fields indicate the ranges of values $l_{\mathrm{ch}}$ values for $q$-SIAs $(q=1-5)$ in Fe. The dashed lines indicate theoretical sink strengths for 1D and 3D diffusion mechanisms calculated by Eqs. (1) and (2)
As an illustration, if $R=10 a$ and $N=(200 a)^{-3}$, $k_{3 \mathrm{D}}^{2} / k_{\mathrm{ID}}^{2} \approx 1700$.

The sink strength for a mixed 1D/3D defect diffusion can be calculated using the object kinetic Monte Carlo method (OKMC) [27, 28]. Such calculations have been carried out in this study for spherical absorbers with $R=10 a$ and $N=(200 a)^{-3}$ to estimate sink strength $k^{2}$ dependence on $l_{\text {ch }}$ by a technique similar to the techniques proposed in $[27,28]$, but different in the following aspects: 1) migration directions may change at each time step of the algorithm in accor-dance with a given probability for such an event (not every $n$ jumps, where $n$ is a parameter, as in $[27,28]) ; 2$ ) the absorbers were not randomly located in the computational cell as in $[27,28]$ but formed a simple orthorhombic lattice with parameters $125 a, 256 a, 250 a$. Fig. 5 shows the resulting dependence.

$k^{-1}$ is the radiation defects' diffusion length (the average distance that the defects can travel from their origination sites to the sinks where they are absorbed 
or annihilated). Let us denote the diffusion length for the case of fully 3D- and 1D-migrating defects as $k_{3 \mathrm{D}}^{-1}$ and $k_{1 \mathrm{D}}^{-1}$, respectively. For the considered spatial configuration of absorbers, Eqs. (1) and (2) give $k_{3 \mathrm{D}}^{-1}=252 a$ and $k_{\mathrm{ID}}^{-1}=10396 a$, respectively. As one can see from Fig. $5, k^{2} \approx k_{3 \mathrm{D}}^{2}$ at $l_{\mathrm{ch}}=k_{3 \mathrm{D}}^{-1}$. Similarly, $k^{2} \approx k_{1 \mathrm{D}}^{-1}$ at $l_{\mathrm{ch}}$ ? $k_{1 \mathrm{D}}^{-1}$. The OKMC values of $k^{2}$ deviate from $k_{3 \mathrm{D}}^{2}$ by not more than $10 \%$ at $l_{\mathrm{ch}} \leq 10 \mathrm{a}$. Such a criterion can be used to assess the need to account for the contribution of the 1D diffusion mechanism to the diffusion when constructing OKMC models of SIA clusters diffusion. For example, for the discussed configuration of absorbers, one can conclude, using this criterion, that the effect of the 1D diffusion mechanism on the sink strength must be considered for SIA clusters with $q \geq 3$. For single SIAs and 2-SIA clusters, the effect of the 1D mechanism is too small to modify $k^{2}$ in any noticeable way.

In iron, the cluster distributions of SIAs generated in atomic collision cascades depend on the cascades' damage energy [3, 4]. Correspondingly, neutron damage irradiations with different neutron energy spectra generate different cluster distributions of SIAs. As the sink strengths of various microstructure elements for radiation defects are sensitive to radiation defects diffusion mechanism, the difference of the fluxes of radiation defects of different signs (vacancies, SIAs) to the microstructure elements is determined not only by the generation rate for radiation defects (dpa) but also by the cluster distribution of SIAs specific for the given neutron spectrum.

\section{CONCLUSIONS}

MD simulation of diffusion trajectories of the SIA clusters (clusters of three, four and five SIAs) has been performed for a temperature range of $300-1200 \mathrm{~K}$ in bcc Fe. Temperature dependences of diffusion characteristics (diffusivity $D^{\mathrm{d}}$, tracers correlation factor $f^{\mathrm{tr}}$, frequency of migration direction changes $v^{\mathrm{R}}$, average distance traveled between migration direction changes $l_{\text {ch }}$ ) for single SIAs and SIA clusters containing up to 5 SIAs have been obtained based on MD data from this study and previous research. Their diffusion activation energies $E^{\mathrm{AD}}$ and reorientation activation energies $E^{\mathrm{R}}$ have been also determined.

At $T \geq 700 \mathrm{~K}, D^{\mathrm{d}}$ for single SIAs and SIA clusters differs by $30 \%$ at most. The $E^{\mathrm{AD}}$ values for all types of SIA clusters $(0.025-0.051 \mathrm{eV})$ are $2-4$ times lower than those for single SIAs $(0.101 \mathrm{eV})$. At room temperature, the difference of $D^{\mathrm{d}}$ values for different defect types can exceed an order of magnitude. At $T \leq 400 \mathrm{~K}, D^{\mathrm{d}}$ weakly depends on $T$ for 3-SIA and 5-SIA clusters $\left(E^{\mathrm{AD}} \leq 0.01 \mathrm{eV}\right)$. The $E^{\mathrm{AD}}$ values for SIA clusters vary from $0.004 \mathrm{eV}$ for 3-SIA clusters to $0.09 \mathrm{eV}$ for 4-SIA clusters, which is significantly lower than the value for single SIAs $(0.24 \mathrm{eV})$.

Single SIAs show a predominantly $3 \mathrm{D}$ diffusion at temperatures $250-1200 \mathrm{~K}$, with $l_{\mathrm{ch}} \approx 2.1 a$. For 2-SIA clusters, the effect of mixed 1D/3D diffusion is most pronounced, with $l_{\mathrm{ch}}$ varying from $7.2 a$ to $10.6 a$ at temperatures $250-1000 \mathrm{~K}$. The $1 \mathrm{D}$ diffusion mechanism prevails for 3-, 4-, 5-SIA clusters: $l_{\mathrm{ch}}$ varies from $283 a$ to $10.7 a$ for 3 -SIA clusters, from $1051 a$ to $33.5 a$ for 4 -SIA clusters, and from $1535 a$ to $48.7 a$ for 5 -SIA clusters when $T$ rises from $300 \mathrm{~K}$ to $1200 \mathrm{~K}$. The $E^{\mathrm{R}}$ values are temperature sensitive: $E^{\mathrm{R}} \approx 0.2 \mathrm{eV}$ for clusters of three to five SIAs at $T<700-800 \mathrm{~K} ; E^{\mathrm{R}} \approx 0.4 \mathrm{eV}$ for 3 -SIA clusters, $E^{\mathrm{R}} \approx 0.9 \mathrm{eV}$ for 4 -SIA, $E^{\mathrm{R}} \approx 1.13$ for 5 -SIA clusters at $T>800 \mathrm{~K}$.

The dependence of diffusion mechanism of SIA clusters on the number of their constituent SIAs together with the dependence of the cluster distributions of SIAs on the atomic collision cascades damage energy and the sensitivity of the sink strengths of microstructure elements to the radiation defects diffusion mechanism cause the evolution of the radiation microstructure under irradiation in different neutron spectra to be different at the same damaging doses (dpa) which must be taken into account when analyzing and interpreting the results of radiation tests of materials of nuclear fission and fusion energy.

The study was supported by NRC «Kurchatov Institute» (grant № 1934a from 28.09.2020) and carried out using computing resources of the Federal collective usage center Complex for Simulation and Data Processing for Mega-science Facilities at NRC «Kurchatov Institute», http://ckp.nrcki.ru/. 


\section{REFERENCES}

1. Indenbom V.L., Lothe J. (Eds.) Elastic Strain Fields and Dislocation Mobility. — Netherlands: North-Holland, 1992.

2. Stoller R.E., Zarkadoula E. 1.20 - Primary Radiation Damage Formation in Solids. — Comprehensive Nuclear Materials (Second Edition), Elsevier, 2020, vol. 1, p. 620 — 662, https://doi.org/10.1016/B978-0-12-803581-8.00661-5.

3. Sivak A.B., Demidov D.N., Zolnikov K.P., Korchuganov A.V., Sivak P.A., Romanov V.A., Chernov V.M. The primary radiation damage in bcc metals Fe and V: analysis of molecular dynamic data. - VANT. Ser. Materialovedenie i Novye Materialy, 2019, vol. 4(100), p. 25-57 (in Russian); https://www.elibrary.ru/item.asp?id=44630371.

4. Shpanskiy Yu.S., the DEMO-FNS project team. Progress in the design of the DEMO-FNS hybrid facility. - Nuclear Fusion, 2019, vol. 59, 076014, p. 1-8; https://doi.org/10.1088/1741-4326/ab14a8.

5. Gao F., Bacon D.J., Osetsky Yu.N., Flewitt P.E.J., Lewis T.A. Properties and evolution of sessile interstitial clusters produced by displacement cascades in $\alpha$-iron. - J. Nucl. Mater., 2000, vol. 276, p. 213-220, https://doi.org/10.1016/S0022-3115(99)00180-4.

6. Osetsky Yu.N., Bacon D.J., Serra A., Singh B.N., Golubov S.I. One-dimensional atomic transport by clusters of self-interstitial atoms in iron and copper. — Philos. Mag., 2003, vol. 83, p. 61—91, https://doi.org/10.1080/0141861021000016793.

7. Gao F., Heinisch H., Kurtz R.J., Osetsky Yu.N. Migration and directional change of interstitial clusters in $\alpha$-Fe: searching for transition states by the dimer method. — Philos. Mag., 2005, vol. 85, p. 619—627, https://doi.org/10.1080/02678370412331320062.

8. Romanov V.A., Sivak A.B., Chernov V.M. Crystallographic, energetic and kinetic properties of self-point defects and their clusters in bcc-iron. 5. Self-interstitial atoms. - VANT. Ser. Materialovedenie i Novye Materialy, 2006, vol. 1(66), p. 202 -222 (in Russian), https://www.elibrary.ru/item.asp?id=22614324.

9. Romanov V.A., Sivak A.B., Chernov V.M. Crystallographic, energetic and kinetic properties of self-point defects and their clusters in bcc-iron. 6. Clusters of self-interstitial atoms. - VANT. Ser. Materialovedenie i novye materialy, 2006, vol. 1(66), p. 223-232 (in Russian), https://www.elibrary.ru/item.asp?id=22614325.

10. Terentyev D.A., Klaver T.P.C., Olsson P., Marinica M.-C., Willaime F., Domain C., Malerba L. Self-trapped interstitial-type defects in iron. - Phys. Rev. Letters, 2007, vol. 100, 145503, p. 1-7, https://doi.org/10.1103/PhysRevLett.100.145503.

11. Terentyev D.A., Malerba L., Hou M. Dimensionality of interstitial cluster motion in bcc-Fe. — Phys. Rev., 2007, vol. 75, 104108, p. 1-13, https://doi.org/10.1103/PhysRevB.75.104108.

12. Sivak A.B., Romanov V.A., Chernov V.M. Diffusion characteristics of self-point defects in bcc iron. - Perspektivnye Materialy, 2009, № 6, p. 5-11 (in Russian), https://elibrary.ru/item.asp?id=12975968.

13. Sivak A.B., Romanov V.A., Chernov V.M. Diffusion of self-point defects in body-centered cubic iron crystal containing dislocations. - Crystal. Rep., 2010, vol. 55, p. 97-108, https://doi.org/10.1134/S1063774510010153.

14. Anento N., Serra A., Osetsky Yu.N. Atomistic study of multimechanism diffusion by self-interstitial defects in $\alpha$-Fe. — Modelling Simul. Mater. Sci. Eng., 2010, vol. 18, 025008, p. 1-18, https://doi.org/10.1088/0965-0393/18/2/025008.

15. Malerba L., Ackland G.J., Becquart C.S., Bonny G., Domain C., Dudarev S., Fu C.-C., Hepburn D., Marinica M.C., Olsson P., Pasianot R.C., Raulot J.M., Soisson F., Terentyev D., Vincent E., Willaime F. Ab initio calculations and interatomic potentials for iron and iron alloys: Achievements within the Perfect Project. - J. Nucl. Mater., 2010, vol. 406, p. 7-18, https://doi.org/10.1016/j.jnucmat.2010.05.016.

16. Ryabov V.A., Pechenkin V.A., Molodtsov V.L., Terentyev D. Contribution of di-SIA to mass transport in $\mathrm{Fe}-\mathrm{Cr}$ alloys. $-\mathrm{J}$. Nucl. Mater., 2016, vol. 472, p. 43—46, https://doi.org/10.1016/j.jnucmat.2016.01.025.

17. Demidov D.N., Sivak A.B., Sivak P.A. Thermal dissociation of di-interstitials in bec Fe and V: a molecular dynamics study. PAS\&T. Ser. Thermonuclear Fusion, 2019, vol. 42, p. 99-107 (in Russian), https://doi.org/10.21517/0202-3822-2019-42-2-99-107.

18. Demidov D.N., Sivak A.B., Sivak P.A. Crystallographic, energetic and diffusion characteristics of di-interstitials in bcc metals Fe and V. — PAS\&T. Ser. Thermonuclear Fusion, 2019, vol. 42, p. 85-96 (in Russian), https://doi.org/10.21517/0202-3822-2019-423-85-96.

19. Sivak A.B., Romanov V.A., Demidov D.N., Sivak P.A., Chernov V.M. Interatomic interaction potentials for simulation of atomic collision cascades and self-point defects in bcc Fe and V metals. — VANT. Ser. Materialovedenie i Novye Materialy, 2019, vol. 4(100), p. 5-24 (in Russian), https://www.elibrary.ru/item.asp?id=44630370.

20. Sivak A.B., Romanov V.A., Chernov V.M. Influence of stress fields of dislocations on formation and spatial stability of point defects (elastic dipoles) in anisotropic bcc crystals of vanadium and iron. - VANT. Ser. Materialovedenie i Novye Materialy, 2002, vol. 1(59), p. 29-40 (in Russian).

21. Sivak A.B., Romanov V.A., Chernov V.M. Influence of stress fields of dislocations on formation and spatial stability of point defects (elastic dipoles) in $\mathrm{V}$ and $\mathrm{Fe}$ crystals. - J. Nucl. Mater., 2003, vol. 323, p. 380-387, https://doi.org/10.1016/j.jnucmat.2003.08.014.

22. Romanov V.A., Sivak A.B., Chernov V.M. Crystallographic, energetic and kinetic properties of self-point defects and their clusters in bcc-iron. 1. Semi-empirical model of bcc iron and interatomic interaction potential. - VANT. Ser. Materialovedenie i Novye Materialy, 2006, vol. 1(66), p. 129—150 (in Russian), https://www.elibrary.ru/item.asp?id=22614316.

23. Romanov V.A., Sivak A.B., Chernov V.M. Crystallographic, energetic and kinetic properties of self-point defects and their clusters in bcc-iron. 2. Basic relations for calculations of defect characteristics and testing results for applied model. - VANT. Ser. Materialovedenie i Novye Materialy, 2006, vol. 1(66), p. 151-171 (in Russian), https://www.elibrary.ru/item.asp?id=22614317.

24. Verlet L. Computer «experiments» on classical fluids. I. Thermodynamical properties of Lennard-Jones molecules. — Phys. Rev., 1967, vol. 159, p. 98-103, https://doi.org/10.1103/PhysRev.159.98.

25. Allnatt A.R., Lidiard A.B. Atomic Transport in Solids. — Cambridge University Press, 1993.

26. Manning J.R. Diffusion Kinetics for Atoms in Crystals. — Toronto, Canada: D. Van Nostrand Company, 1968. 
27. Heinisch H.L., Singh B.N., Golubov S.I. A kinetic Monte Carlo study of mixed 1D/3D defect migration. - J. Comput. Aided Mater. Des., 1999, vol. 6, p. 277-282, https://doi.org/10.1023/A:1008777901639.

28. Malerba L., Becquart C.S., Domain C. Object kinetic Monte Carlo study of sink strengths. — J. Nucl. Mater., 2007, vol. 360, p. 159-169, https://doi.org/10.1016/j.jnucmat.2006.10.002.

29. Osetsky Yu.N., Mikhin A.G., Serra A. Study of copper precipitates in $\alpha$-iron by computer simulation. I. Interatomic potentials and properties of Fe and Cu. — J. Nucl. Mater., 1994, vol. 212 - 215, p. 236-240, https://doi.org/10.1080/01418619508239930.

30. Schultz F. Atomic defects in metals Fe. - In: Ullmaier H. (ed.), Atomic Defects in Metals. Landolt-Börnstein - Group III Condensed Matter. Berlin: Springer-Verlag, 1991, vol. 25, p. 125—134, https://doi.org/10.1007/10011948_46.

31. Wiedersich H. On the theory of void formation during irradiation. — Radiat. Eff., 1972, vol. 12, p. 111-125, https://doi.org/10.1080/00337577208231128.

32. Brailsford A.D., Matthews J.R., Bullough R. The effect of recombination on sink strengths in the rate theory of void-swelling. J. Nucl. Mater., 1979, vol. 79, p. 1-13, https://doi.org/10.1016/0022-3115(79)90428-8.

33. Barashev A.V., Golubov S.I., Trinkaus H. Reaction kinetics of glissile interstitial clusters in a crystal containing voids and dislocations. — Philos. Mag. A, 2001, vol. 81, p. 2515—2532, https://doi.org/10.1080/01418610108217161.

\section{AUTHORS}

Alexander Borisovich Sivak, PhD., head of laboratory; NRC «Kurchatov Institute», 1, Academika Kurchatova sq., Moscow, 123182, Russia, Sivak_AB@nrcki.ru

Dmitry Nikolaevich Demidov, PhD. student, research engineer; NRC «Kurchatov Institute», 1, Academika Kurchatova sq., Moscow, 123182, Russia, Demidov_DN@nrcki.ru

Polina Alexandrovna Sivak, engineer; NRC «Kurchatov Institute», 1, Academika Kurchatova sq., Moscow, 123182, Russia, Sivak_PA@nrcki.ru

Received 15 January 2021

Revised 16 March 2021

Accepted 25 March 2021

Problems of Atomic Science and Technology Ser. Thermonuclear Fusion, 2021, vol. 44, issue 2, pp. 148-157 